\title{
A Study of Incidence and outcome of Peripartum Cardiomy- opathy in a Tertiary Care Teaching Hospital
}

\author{
Kanaparthi Spandana ${ }^{1}$, Suresh Jana ${ }^{2}$, Kasturi Ravinder Reddy ${ }^{3}$, Mudgalkar Nikhil ${ }^{4}$
}

1. Post graduate, Department of Medicine,Prathima Institute of Medical Sciences, Nagnur Road,Karimnagar

2. Assistant Professor, Department of General Medicine, Prathima Institute of Medical Sciences, Naganoor, Karimnagar.

3. Professor and Head ,Department of General Medicine and consultant Cardiologist,Prathima Institute of Medical Sciences, Nagnur Road, Karimnagar

4. Professor , Department of Anaesthesia, Prathima Institute of Medical Sciences,Nagnur Road,Karimnagar

Corresponding Author:Dr. Suresh Jana, H. No. 3-1-342, Ambedkar Road, Old Employment office, Karimnagar 505001. Email: drusresh2k2@gmail.com Mobile: 7799270000

Submission: 20/12/2020

Review: 1/1/2021

Acceptance: 10/1/2021

DOI:10.47799/pimr.0902.13

\section{ABSTRACT}

Background: Heart failure in women associated with pregnancy and the peripartum period is now recognized as a distinctive form of cardiomyopathy. Its prevalence and mortality vary in different geographical locations. There is a paucity of data from our group of the population regarding peripartum cardiomyopathy hence we conducted the study to determine the incidence, aetiology of PPCM in our study group along with treatment and maternal outcomes.

Methods: Patients with any parity and age, which are in their peripartum period i.e. one month before delivery or within five months of delivery. Patients presenting with moderate to severe breathlessness at rest or on exertion with palpitations, ankle oedema, or with signs and symptoms of heart failure. Documented systolic dysfunction with the echocardiographic finding of Ejection fraction of $<45 \%$ and or Fractional shortening $<30 \%$, absence of another identifiable cause for the HF.

Results: Overall incidence of PPCM in our institution is 9 per 1000 deliveries. Among $n=67$ PPCM cases, $n=26$ patients (38.80\%) developed PPCM during pregnancy and $n=41$ patients (61.2\%) during postpartum period. The majority of the patients $\mathrm{n}=49$ cases $(73.13 \%)$ had severe left ventricular systolic dysfunction ( $E F=30 \%)$ and $n=10(14.92 \%)$ had moderate left ventricular systolic dysfunction and $n=8$ had mild LV dysfunction. There was no significant statistical difference in the severity of LV dysfunction between primi and multiparous women.
Conclusion: The main factors are advanced maternal age and multiparity. Twin pregnancies are also risk factors for the development of PPCM. Yet another important risk factor was Pre-eclampsia. There is no single explanation for the pathogenesis of PPCM that is relevant for all women. Recognition of symptoms of breathlessness, orthopnea, and pedal edema in the post-partum period with a history of preeclampsia should arouse a high degree of suspicion.

Keywords: Peripartum Cardiomyopathy [PPCM], Incidence, Heart failure, cardiomyopathy

\section{INTRODUCTION}

Peripartum cardiomyopathy (PPCM) is a rare and potentially life-threatening form of heart failure affecting women late in pregnancy or the early puerperium. There is no single explanation for the pathogenesis of PPCM. It is a diagnosis of exclusion. A high index of suspicion is required for the diagnosis, as shortness of breath and pedal oedema are common in the peripartum period. Peripartum cardiomyopathy is a distinct form of cardiomyopathy, associated with high morbidity and mortality, but also with the possibility of full recovery. Heart failure associated with pregnancy and the peripartum period was recognized in

the literature as early as the 1800 s by Virchow and others.[1]PPCM was first recognized as a distinctive form of cardiomyopathy in 1937, when Gouley et al noticed features of severe heart failure with an atypical and distinct dilated cardiomyopathy in later months of pregnancy in 7 pregnant 
females, which persisted after delivery. ${ }^{[2]}$ Earlier terms for this condition include toxic postpartum HF,Meadows' syndrome, Zaria syndrome, and postpartum myocarditis. A large prospective international registry of 411 women from 43 countries has demonstrated that PPCM occurs globally, affecting women from all ethnicities on all continents. ${ }^{[3]}$ However, incidence rates vary widely depending on geographical location. ${ }^{[4-7]}$ Despite many attempts to uncover a distinct etiology of PPCM, the cause remainsunknown and may be multifactorial. Experimental research suggests that these multiple factors result in a common final pathway with enhanced oxidative stress, cleavage of prolactin to an angiostatic $\mathrm{N}$-terminal $16 \mathrm{kDa}$ prolactin fragment, and impaired vascular endothelial growth factor (VEGF) signaling because of upregulated soluble forms like tyrosine kinase (sFLT1). ${ }^{8-}$ ${ }^{10]}$ The precise incidence in India is not known, an incidence of one case per 1374 live births has been reported from a tertiary care hospital from South India. $\left.{ }^{[11}\right]$ This study gains its importance as there are major discrepancies in the reported incidence and mortality rates of PPCM, Such discrepancies most likely arise from the lack of prospective PPCM registries worldwide, and as a result, optimal treatment strategies and definitive predictors of left ventricular recovery associated with PPCM remain unknown and as peripartum cardiomyopathy seems to affect women in different parts of the world but with considerable differences in clinical presentation.

\section{Material and methods}

This retrospective study was conducted in the Department of OBGY and Cardiology, Prathima Institute of Medical Sciences, Karimnagar. Retrospective data were collected from hospital records from January2015 to October 2018.

Institutional Ethical committee permission was obtained for the study. Written consent was obtained from all patients of the study.

Inclusion Criteria:

1. Patients with any parity and age, who are in their peripartum period i.e. one month before delivery or within five months of delivery.

2. Patients presenting with moderate to severe breathlessness at rest or on exertion with palpitations, ankle oedema, or with signs and symptoms of heart failure.

3. Documented systolic dysfunction with the echocardiographic finding of

4. Ejection fraction of $<45 \%$ and or

5. - Fractional shortening $<30 \%$

6. Absence of another identifiable cause for the HF.

\section{Exclusion Criteria:}

1. Patients with pre-existing cardiomyopathy(idiopathic dilated cardiomyopathy,

2. Familial dilated cardiomyopathy or HIV/AIDS cardiomyopathy).

3. Patients with pre-existing acquired or congenital valvular heart disease.

4. Patients with pre-existing undetected congenital heart disease.

5. Patients with diastolic heart failure due to hypertensive heart disease(Chronic

6. Hypertensive patients).

7. Patients with myocardial infarction secondary to coronary artery dissection,

8. coronary artery disease, coronary embolus/thrombosis (in a normal coronary

9. Artery), and coronary artery spasm.

10. Patients with pulmonary embolism/ amniotic fluid embolism, chronic obstructive

11. airway disease, severe anemia(hemoglobin less than $10 \mathrm{~g} /$ dl), pulmonary artery

12. Hypertension either primary or secondary, thyroid disorders, septicemia.

\section{Patients with normal echocardiography.}

Based on the inclusion and exclusion criteria $n=67$ cases were taken for study.

1. All the selected patients' detailed history including mode of delivery, complications, and maternal as well as the fetal outcome and details of complete physical examination and data was collected in a pre-designed proforma.

2. Patients' investigations records which included Complete blood count, ESR, Fasting Blood sugar, Serum electrolytes, Lipid profile, Electrocardiography, Echocardiography, HIV serology was collected.

3. Each patient has followed up examination reports for a period of 1 year was recorded including her ECG, echocardiographic and clinical findings.

4. Retrospective data of old cases were collected from hospital records.

5. Statistical analysis was done using SPSS version 19 - Student unpaired T-Test

Results:

A total of $n=6880$ cases were delivered from January 2015 to October 2018. N=67 PPCM cases were identified among them. 
$\mathrm{N}=21$ PPCM cases were identified out of $n=2230$ deliveries in 2015. Out of $n=2010$ deliveries, $n=18$ cases had PPCM. $N=15$ PPCM patients were identified among $n=1800$ deliveries in 2017. In 2018, n=840 cases were delivered till October. Among them, $n=13$ cases were PPCM. The overall incidence of PPCM in our institution is 9 per 1000 deliveries. $\mathrm{N}=53$ patients were

\begin{tabular}{|c|c|c|}
\hline Age Groups & Primiparous & Multiparous \\
\hline $20-25$ & 31 & 2 \\
\hline $26-30$ & 19 & 9 \\
\hline $31-35$ & 5 & 1 \\
\hline
\end{tabular}

Among $n=67$ PPCM cases, $n=26$ patients (38.80\%) developed PPCM during pregnancy and $n=41$ patients $(61.2 \%)$ during postpartum period. Most of the patients developed PPCM in the first month following delivery. Presentation after delivery ranged from two days to two months. All patients presented with symptoms and signs of heart failure $n=12$ had preeclampsia and 2 had eclampsia.

The majority of the patients $n=49$ cases $(73.13 \%)$ had severe

left ventricular systolic dysfunction ( $E F=30 \%$ ) and $n=10$ (14.92\%) had moderate left ventricular systolic dysfunction and $\mathrm{n}=8$ had mild LV dysfunction. There was no significant statistical difference in the severity of LV dysfunction between primi and multiparous women. And, there was no significant statistical difference in the severity of LV dysfunction between young and elderly mothers.

Table 2: Parity and severity of Left ventricular dysfunctions

\begin{tabular}{|c|c|c|c|c|}
\hline & Primi & Multi & T-test & \multirow{2}{*}{0.84419} \\
\cline { 1 - 3 } No. of cases & 55 & 31.83 & $>0.4155$ \\
Mean EF & 29.21 & 7.41 & \\
\hline
\end{tabular}

$\mathrm{N}=52$ patients had a normal delivery and $\mathrm{n}=15$ patients underwent caesarean section. Indication for caesarean section in all the cases was an obstetric cause (Breech presentation, elective repeat caesarean section, cephalopelvic disproportion, and severe oligohydramnios). There were two intrauterine fetal deaths and $n=1$ neonatal death. Two cases developed CVA and one developed cerebral sinus venous thrombosis and two developed pneumonia. Two were complicated with Torsades de pointes. There were $n=6$ maternal deaths (8.9\%). Recovery of LV Function among remaining $n=61$ cases, EF improved within $n=2$ months in $n=32$ cases $(55.17 \%)$, within 3 months in $\mathrm{n}=16$ cases $(27.58 \%)$ and $\mathrm{n}=10$ had persistent $\mathrm{LV}$ dysfunction (17.25\%), and $n=3$ cases lost to follow-up.

\section{Discussion}

Peripartum cardiomyopathy (PPCM) is one of the causes of pregnancy-associated heart failure. It typically develops during the last month of and up to 5 months after, pregnancy in women without known cardiovascular disease. A high index of suspicion is required for the diagnosis, as shortness of breath and pedal oedema are common in the peripartum period. Although the disease is relatively uncommon, its incidence is increasing. The incidence of PPCM varies extensively across geographic regions of the world from 1:15,000 to1:100 deliveries 20. The precise incidence in India is not known. There are major discrepancies in the reported incidence and mortality rates of PPCM.

The incidence in the present study was 9 cases per 1000 deliveries. Vinay $\mathrm{P}$ et $\mathrm{al}^{;}{ }^{[11]}$ in South India have reported an incidence of 1 case per 1374 deliveries. Joshi AV et al; ${ }^{[12]}$ reported 22 per 10279 deliveries in study conduct in Maharashtra.Fett JD et al; ${ }^{[13]}$ reported a higher incidence of one case per 300 live births from Haiti. Mielniczuk et al; [14] reported an incidence of one case for 3189 live births in the USA. Lee et al; ${ }^{[15]}$ reported 1 per 1741 deliveries in a study conducted in South Korea.Chee $\mathrm{KH}$ et al; ${ }^{[16]}$ reported a prevalence of 34 per 100,000 live births in Malaysia. In this study, among $n=67$ PPCM cases, $n=26$ patients $(38.80 \%)$ developed PPCM during pregnancy and $n=41$ patients $(61.2 \%)$ during postpartum period. Most of the patients developed PPCM in the first month following delivery. Peripartum stress due to altered physiological conditions might be the cause behind PPCM in the early puerperium. Laghari $\mathrm{AH}$ et al; 
${ }^{[17]}$ found out of 45 cases, $14(31.1 \%)$ presented during pregnancy, and 31 (68.8\%) presented after delivery. In a study by Lampert LB et al;[18]78\% of cases occurred during the postpartum period. PPCM is thought to be more prevalent in the upper and lower extremes of childbearing age, and in older women of high parity, it is important to note that $24-37 \%$ of cases may occur in young primigravid patients. ${ }^{[13,19]}$ Several cases from Nigeria, Haiti, and South Africa did not show a disproportionate role for older age and multiparity in the development of PPCM. ${ }^{[20]}$ Elkayam et al; ${ }^{[21]}$ study did not support a strong association between multiparity and development of PPCM because almost $40 \%$ of the cases occurred in association with their first pregnancy. In this study, the mean ejection fraction at the time of presentation was $30.25 \pm 6.05$.The mortality rate was higher among the patients with severe LV dysfunction, while most of the survivors had mild to moderate LV dysfunction which was statistically significant $(p<0.005)$ Majority of the patients i.e. $n=49$ cases (73.13\%) had severe left ventricular systolic dysfunction (EF $=30 \%)$ and $10(14.93 \%)$ had moderate left ventricular systolic dysfunction and 8 (11.94\%) had mild LV dysfunction. The mean Ejection fraction at the time of presentation was $25.3 \pm 9.8 \%$ in a study by Joshi AV et al; ${ }^{[12]}$ n a study by Laghari AH et al; ; ${ }^{[17]}$ out of 45 cases, 39 (86.6\%) had severe LV systolic dysfunction at presentation and 6 (13.3\%) had moderate LV systolic dysfunction. Many PPCM studies purposefully excluded women with Pre-eclampsia or eclampsia to avoid misclassification of Pre-Eclampsia associated pulmonary edema as PPCM.Bello et al; ${ }^{[22]}$ recommended that women with Pre-Eclampsia are not to be excluded from future studies of PPCM, considering their strong association. As blood volume expansion and other hemodynamic changes are greater in twin or multifetal pregnancies than singleton pregnancies, cardiac overload may be more likely to occur and the serum precursor protein BNP (NT-proBNP) level in the late stage of pregnancy is significantly higher in twin than singleton pregnancies. Women with multifetal or twin pregnancies are at a markedly elevated risk of developing PPCM. In this study, there were only two twin pregnancies $(2.9 \%)$. In a study by Chapa et al; ; ${ }^{[23]}$ four women $(12.5 \%)$ out of 32 cases had multifetal gestations ( 3 twins, 1 triplet). In a study by Hasan et al; ${ }^{[24]} 2(6.25 \%)$ out of 32 patients had multifetal pregnancies. In the study by Vinay et al; ${ }^{[11]}$ there were no twin pregnancies among 9 PPCM cases. $6.6 \%$ of patients had twin pregnancy in a study by Laghari $\mathrm{AH}$ et al; ${ }^{[17]}$ Twin or triplet pregnancies were reported in $15 \%$ in the study byGoland et al; ${ }^{[25]}$ out of 182 patients. Sliwa et al; ${ }^{[26]}$ suggest that beta-blockers should be continued for at least 1 year. In the present study, all the patients have been managed with inotropic support, intravenous diuretics ACE inhibitors (in postpartum cases), and beta-blockers.Laghari AH et al; ${ }^{[17]}$ three out of 40 patients (6.6\%) had ventricular tachycardia (VT) at presentation. The prognosis in PPCM varies geographically. In the United States, reported mortality rates associated with PPCM have varied widely between $0 \%$ and $19 \% .{ }^{[27]}$ In the present study, there were 6 (8.95\%)maternal deaths. 5 patients were primiparous and one was multiparous. All 6 cases were aged less than 30 years and had severe LV dysfunction.

\section{Conclusion}

Within the limitations of the current study, we found the incidence of PPCM in our population to be 9 per 1000 deliveries. The main factors are advanced maternal age and multiparity. Twin pregnancies are also risk factors for the development of PPCM. Yet another important risk factor was Pre-eclampsia. There is no single explanation for the pathogenesis of PPCM that is relevant for all women. Recognition of symptoms of breathlessness, orthopnea and pedal oedema in the post-partum period with a history of preeclampsia should arouse a high degree of suspicion. Early diagnosis and treatment lead to good maternal and foetal outcomes.

\section{REFERENCES}

1. Ritchie C. Clinical contributions to the pathology, diagnosis, and treatment of certain chronic diseases of the heart. John Stark; 1850.

2. Gouley BA. Idiopathic myocardial degeneration associated with pregnancy and especially the peripartum. Am J Med Sci. 1937;19:185-99.

3. Liwa K, Mebazaa A, Hilfiker-Kleiner D, Petrie MC, Maggioni $A P$, et al. Clinical characteristics of patients from the worldwide registry on peripartum cardiomyopathy (PPCM) EURObservational Research Program in conjunction with the Heart Failure Association of the European Society of Cardiology Study Group on PPCM. European journal of heart failure. 2017;19(9):1131-41.

4. Sliwa, K., Hilfiker-Kleiner, D Petrie, M.C., Mebazaa, A., et al. Current state of knowledge on aetiology, diagnosis, management, and therapy of peripartum cardiomyopathy: a position statement from the Heart Failure Association of the European Society of Cardiology Working Group on peripartum cardiomyopathy. European journal of heart failure 2010;12(8):767-78.

5. Pearson GD, Veille JC, Rahimtoola S, Hsia J, Oakley CM, Hosenpud JD, Ansari A, Baughman KL. Peripartum cardiomyopathy: national heart, lung, and blood institute and office of rare diseases (national institutes of health) workshop recommendations and review. Jama. 2000 Mar 1;283(9):1183-88.

6. Seftel $H$, Susser M. Maternity and myocardial failure in African women. Br Heart J 1961; 23:43. 
7. Davidson NM, Parry EH. The etiology of peripartum cardiac failure. Am Heart J 1979; 97:535.

8. Woolford RM. Postpartum myocardosis. Ohio State Med J 1952; 48:924.

9. Pierce JA, Price BO, Joyce JW. Familial occurrence of postpartal heart failure. Arch Intern Med 1963; 111:651.

10. Sliwa K, Damasceno A, Mayosi BM. Epidemiology and etiology of cardiomyopathy in Africa. Circulation 2005; 112:3577.

11. Pandit V, Shetty S, Kumar A, Sagir A. Incidence and outcome of peripartum cardiomyopathy from a tertiary hospital in South India. Tropical doctor. 2009;39(3):16869.

12. Aditi V. Joshi, Michelle N. Fonseca, Deepali S. KharatKapote. A study of peripartum cardiomyopathy in a tertiary care center in India. Int J Reprod Contracept Obstet Gynecol. 2017 Feb;6(2):523-526.

13. Fett JD, Christie LG, Carraway RD, Murphy JG. Five-year prospective study of the incidence and prognosis of peripartum cardiomyopathy at a single institution. Mayo Clin Proc 2005; 80:1602.

14. Mielniczuk LM, Williams K, Davis DR, et al. Frequency of peripartum cardiomyopathy.Am J Cardiol 2006;97:176568.

15. Lee S, Cho GJ, Park GU, Kim LY, Lee TS, Kim DY, Choi SW, Young JC, Han SW, Ryu KH, NaJO. Incidence, risk factors, and clinical characteristics of peripartum cardiomyopathy in SouthKorea. Circulation: Heart Failure. 2018;11(4):e004134.

16. Chee $\mathrm{KH}$, Azman W. Prevalence and outcome of peripartum cardiomyopathy in Malaysia. Int J Clin Pract 2009;63:722-25.

17. Laghari, A.H., Khan, A.H. Kazmi, K.A. Peripartum cardiomyopathy: ten-year experience at a tertiary care hospital in Pakistan. BMC Research Notes 2013; 6:495.

18. Lampert MB, Lang RM. Peripartum cardiomyopathy. Am Heart J 1995; 130:860.

19. Desai D, Moodley J, Naidoo D. Peripartum cardiomyopathy: experiences at King Edward VIII Hospital, Durban, South Africa and a review of the literature. Trop Doct 1995;25:118.

20. Karaye KM, Sani MU. Factors associated with poor prognosis among patients admitted with heart failure in a Nigerian tertiary medical centre: a cross-sectional study. BMC Cardiovasc Disord 2008;8(1):16.

21. Elkayam $U$, Akhter MW, Singh $H$, et al. Pregnancyassociated cardiomyopathy: clinicalcharacteristics and a comparison between early and late presentation. Circulation 2005; 111:2050.

22. Bello N, Rendon IS, Arany Z. The relationship between pre-eclampsia and peripartum cardiomyopathy: A systematic review and meta-analysis. J Am Coll Cardiol 2013; 62:1715-23

23. Chapa JB, Heiberger HB, Weinert L, et al. Prognostic value of echocardiography in peripartum cardiomyopathy. ObstetGynecol 2005; 105:1303-08.

24. Mahmood UI Hassan, Bakhatawar Shah, Bushra Rauf, Cheragh Hussain.Peripartum Cardiomyopathy - An InHospital Study. Pak Heart J 2019;52(3):219-223.

25. Goland S, Modi K, Bitar F, et al. Clinical profile and predictors of complications in peripartum cardiomyopathy. J Card Fail 2009; 15:645-50.

26. Sliwa K, Fett J, Elkayam U. Peripartum cardiomyopathy. Lancet 2006;368:68793.

27. Elkayam U. Clinical characteristics of peripartum cardiomyopathy in the United States:diagnosis, prognosis, and management. J Am Coll Cardiol 2011; 58:659-63.

How to cite this article : Kanaparthi S, Jana S, Kasturi RR, Mudgalkar N. A Study of Incidence and outcome of Peripartum Cardiomyopathy in a Tertiary Care Teaching Hospital. Perspectives in Medical Research 2021; 9 (2):58-62

DOI:10.47799/pimr.0902.13

Sources of Support: Nil, Conflict of interest: None declared 